\title{
Utility of computed tomography perfusion in detection of cerebral vasospasm in patients with subarachnoid hemorrhage
}

\author{
Roham Moftakhar, M.D., Howard A. Rowley, M.D., Aquilla Turk, D.O., \\ David B. Niemann, M.D., Beverly AagaARd Kienitz, M.D., \\ Jamie Van Gomple, M.D., AND Mustafa K. BaşKaya, M.D.
}

Departments of Neurosurgery and Radiology, University of Wisconsin Hospital and Clinics, Madison, Wisconsin

\begin{abstract}
Object. Digital subtraction (DS) angiography is the gold standard for detecting cerebral vasospasm after subarachnoid hemorrhage (SAH). Computed tomography (CT) perfusion is a recently developed modality for the evaluation of cerebral hemodynamics. This study was conducted to evaluate the potential of using CT perfusion to detect vasospasm in patients with SAH.

Methods. Fourteen patients between the ages of 41 and 66 years with aneurysmal SAH underwent 23 CT perfusion scans for suspected vasospasm. All patients underwent DS angiography within 12 hours of the CT perfusion scans. The presence of vasospasm on CT perfusion images was determined based on qualitative reading using color maps of mean transit time, cerebral blood flow, and cerebral blood volume as criteria. The presence or absence of vasospasm as retrospectively determined using CT perfusion was compared with DS angiography findings.

Of the 23 CT perfusion scans performed, 21 (91\%) were concordant with angiography findings in predicting the presence or absence of vasospasm. In 15 of 23 scans, the presence of vasospasm was detected on CT perfusion scans and confirmed on DS angiography studies. In two cases, vasospasm was revealed on DS angiography but was not confirmed on CT perfusion. The degree of agreement between CT perfusion and DS angiography for detection of vasospasm was high $(\kappa=0.8, \mathrm{p}<0.0001)$.

Conclusions. Computed tomography perfusion is an accurate, reliable, and noninvasive method to detect the presence or absence of vasospasm. It can be used as a tool to help guide the decision to pursue DS angiography with the intent to treat vasospasm.
\end{abstract}

\section{KEY WORDS • subarachnoid hemorrhage • vasospasm • computed tomography perfusion $\bullet$ cerebral angiography}

$\mathrm{T}$ HE incidence of SAH in the US due to a ruptured intracranial aneurysm is approximately 10 per 100,000 per year. ${ }^{15}$ Delayed cerebral ischemia due to vasospasm occurs in approximately one third of cases and is one of the most devastating sequelae of SAH., ${ }^{910}$ One reason for the high morbidity and mortality rates associated with vasospasm is the lack of modalities that can be used to predict reliably the early onset of cerebral ischemia at a time before the patient suffers permanent neurological deficit. In conscious patients, vasospasm is frequently detected when the individual shows a change in the neurological examination. However, by the time neurological decline is noted, the patient might have suffered irreversible cerebral infarction. In unconscious patients, detection of vasospasm is even more problematic due to our inability to perform a reliable clinical examination. For these

Abbreviations used in this paper: $\mathrm{CBF}=$ cerebral blood flow; $\mathrm{CBV}=$ cerebral blood volume; $\mathrm{CT}=$ computed tomography; $\mathrm{DS}=$ digital subtraction; ICP = intracranial pressure; $\mathrm{PCoA}=$ posterior communicating artery; $\mathrm{SAH}=$ subarachnoid hemorrhage; $\mathrm{TCD}=$ transcranial Doppler. patients, clinicians most often rely on parameters such as vital signs and/or ICP, which can be difficult to interpret. In these circumstances, TCD ultrasonography is a noninvasive modality that is used to detect vasospasm, but it has been shown to have technical limitations, and recent studies have demonstrated a low sensitivity of this modality for diagnosis of cerebral vasospasm., ${ }^{2,12,14}$

Computed tomography perfusion is a CT-based imaging procedure that provides maps of $\mathrm{CBF}, \mathrm{CBV}$, and mean transit time. ${ }^{4,20}$ It has the advantages of being noninvasive, readily available, and providing comprehensive information regarding parenchymal features such as hemorrhage and hydrocephalus. It also could be an option for early detection of cerebral ischemia due to vasospasm. However, the reliability of CT perfusion in the detection of vasospasm has not been examined extensively to date.

The purpose of this study was to determine the reliability of CT perfusion in detecting cerebral ischemia due to vasospasm and to demonstrate the use of this imaging modality in a cohort of patients with a range of Hunt and Hess grades to decide whether DS angiography should be performed. 


\section{Clinical Material and Methods}

\section{Patient Population}

All procedures were performed according to guidelines approved by our institution's internal review board. Between June 2003 and June 2004, 14 patients from 41 to 66 years of age presented with acute aneurysmal SAH and underwent CT perfusion followed within 12 hours by DS angiography. Patients who underwent either CT perfusion without DS angiography or CT perfusion followed by DS angiography more than 12 hours later were excluded from this study.

In the patients included in this study, CT perfusion was performed in three circumstances as follows: 1) at the time of patient presentation as part of the initial baseline imaging workup, followed by DS angiography and then clip occlusion or coil placement for treatment of the aneurysm; 2 ) if vasospasm was suspected, but only when the results of blood gas and chemistry tests and of head CT scans obtained without contrast material could not be used to explain the deterioration in neurological status, changes in vital signs, or elevated ICP; or 3) in unconscious patients in whom a neurological examination could not be performed. For all patients in whom vasospasm was suspected based on CT perfusion scans, DS angiography was performed to help determine a definite diagnosis and treatment plan for the vasospasm. Demographic data for all patients in the study are shown in Table 1.

All of the patients were admitted to the neurosurgical intensive care unit, where they underwent hourly neurological examinations and received prophylactic nimodipine. If indicated, a ventriculostomy was placed to allow for ICP monitoring and cerebrospinal fluid drainage. All patients had a central venous access catheter placed and received intravenous hydration. Every other day, TCD ultrasonography was performed. In patients in whom vasospasm was suspected based on either neurological decline, changes in vital signs, or persistent increase of ICP above $20 \mathrm{~mm} \mathrm{Hg}$, routine laboratory tests to measure arterial blood gas and serum sodium levels were performed to exclude abnormalities that could explain the deterioration. If the test results were normal, a head CT image without addition of contrast material was obtained to rule out hydrocephalus or recurrent hemorrhage.

\section{Perfusion Imaging}

Computed tomography perfusion scans were performed using commercially available 8- or 16-slice scanners (General Electric, Milwaukee, WI). Scanning consisted of an initial head CT study that was obtained without contrast material followed by a dynamic contrast-enhanced CT perfusion acquisition. The perfusion scans were centered at the level of the centrum semiovale. Four contiguous 5$\mathrm{mm}$-thick sections were acquired using a dynamic 40-ml intravenous contrast bolus, scanning for 40 to 45 seconds. Perfusion parameter maps were prepared on a General Electric Advantage Windows Workstation using Perfusion 3 software, version 4.2.

\section{Image Analysis}

All CT perfusion images and DS angiograms were interpreted by experienced senior neuroradiologists. The CT perfusion images were analyzed qualitatively by using rainbow color scale maps of mean transit time, $\mathrm{CBF}$, and $\mathrm{CBV}$. The neuroradiologists who examined the CT perfusion images were not aware of the results of the cerebral angiograms at the time of interpretation. The reports on the CT perfusion images and cerebral angiograms were examined retrospectively, and the presence or absence of cerebral vasospasm as reported was recorded in a database. If the radiology report on either a CT perfusion image or cerebral angiogram did not clearly state the presence or absence of vasospasm, that particular examination was excluded from the analysis.

\section{Statistical Analysis}

To determine the degree of agreement between CT perfusion and DS angiography in detecting vasospasm, а $\kappa$ statistic was used. A commonly used scale for this statistic proposes that a $\kappa$ value of less than 0.2 reflects poor agreement, 0.2 to 0.4 fair agreement, 0.4 to 0.6 moderate agreement, 0.6 to 0.8 substantial agreement, and greater than 0.8 almost perfect agreement.

\section{Results}

A total of $23 \mathrm{CT}$ perfusion scans and corresponding DS angiograms were obtained in 14 patients (Table 2). Of the $23 \mathrm{CT}$ perfusion scans performed, four examinations were part of the initial patient workup on presentation. The other 19 CT perfusion scans were performed because either vasospasm was suspected based on the patient's neurological deterioration or the patient had not undergone a neurological examination. Of the $23 \mathrm{CT}$ perfusion scans performed, 21 (91\%) were confirmed by DS angiography to be correct in predicting the presence or absence of vasospasm. Fifteen of the 23 CT perfusion scans were interpreted as positive for vasospasm, and the corresponding DS angiograms confirmed the presence of vasospasm in all 15 cases. Eight of the $23 \mathrm{CT}$ perfusion scans were interpreted as negative for vasospasm; of these eight scans, six $(75 \%)$ were confirmed by DS angiography as being negative for vasospasm. 


\section{Utility of CT perfusion in detecting cerebral vasospasm after SAH}

fusion and DS angiography in detection of vasospasm was statistically significant $(\mathrm{p}<0.0001)$.

\section{Illustrative Case}

\section{Case 7}

History and Examination. This 61-year-old woman presented with an SAH from a ruptured aneurysm of the right PCoA. She was assigned a clinical Hunt and Hess grade of IV and a Fisher grade of 3.

Operation and Postoperative Evaluation. After imaging, the patient underwent ventriculostomy placement and successful coil embolization of her aneurysm. On the 3rd day after the SAH occurred, she demonstrated a subtle deterioration on neurological examination although her vital signs remained unchanged and her ICP was less than 20 $\mathrm{mm} \mathrm{Hg}$. Due to the lack of an adequate acoustic window, TCD ultrasonography could not be performed. The results of other evaluations, including serum sodium levels, were unremarkable.

Based on her high Fisher grade, it was determined that the patient had a high risk of vasospasm. Because she had a possible subtle decline in her neurological status and TCD ultrasonography was not possible, a head CT scan was obtained without contrast material and a CT perfusion study was performed to help determine whether she should undergo DS angiography. The head CT image did not reveal further hemorrhage or worsening hydrocephalus, but the CT perfusion image demonstrated prolongation of mean transit time along with reduction in $\mathrm{CBV}$ and $\mathrm{CBF}$ in the right hemisphere (Fig. 1). Subsequently, the patient underwent DS angiography, which demonstrated multifocal areas of vasospasm (Fig. 2). The vasospasm was treated using $10 \mathrm{mg}$ of intraarterial verapamil in each vascular territory, which resulted in a subsequent improvement of symptoms.

Postoperative Course and Follow-Up Testing. During her hospital stay, the patient had three other angiograms and four infusions of intraarterial verapamil to treat recurrent vasospasm. Eventually, a tracheostomy and placement of a percutaneous endoscopic gastrostomy tube were per-

FIG. 1. Case 7. Computed tomography perfusion images; perfusion parameter maps demonstrating striking prolongation of mean transit times (MTT), modest reduction in CBV, and mild reduction in CBF in the right hemisphere. 
FIG. 2. Case 7. Angiograms demonstrating multifocal areas of vasospasm, including the supraclinoid carotid artery and anterior cerebral vessel (arrows).

formed, and she was transferred to a rehabilitation facility. On follow-up examination 10 months later, the patient's tracheostomy had been discontinued, she was feeding herself, and she was ambulating with the aid of a walker.

\section{Discussion}

Delayed symptomatic ischemia occurs in 20 to $30 \%$ of patients with SAH.7 Symptomatic ischemia from SAH is associated with a $7 \%$ rate of permanent deficit and a 7\% rate of mortality. In the modern era of SAH management, the morbidity and mortality levels associated with vasospasm have not declined. One of the possible reasons for the failure to improve outcomes is the lack of a practical modality to predict vasospasm accurately before the onset of more pronounced cerebral ischemia and irreversible neuronal damage, at a time when the tissue is salvageable. Therefore, a noninvasive tool that can reliably and accurately detect vasospasm before the onset of permanent deficits is needed.

Several noninvasive modalities are used for the detection of vasospasm. One of the most widely used imaging tools is TCD ultrasonography, which, although practical, faces several limitations. First, it can identify vasospasm with only $67 \%$ specificity compared with that provided by cerebral angiography. ${ }^{12}$ Furthermore, angiography can reliably predict the absence or presence of clinically significant angiographic vasospasm only when there are low flow velocities $(<120 \mathrm{~cm} / \mathrm{sec})$ or very high flow velocities $(\geq 200 \mathrm{~cm} /$ sec). ${ }^{14}$ Additionally, the sensitivity and specificity of TCD ultrasonography is operator-dependent, and only $85 \%$ of patients will have adequate temporal bone windows to allow for the performance of this modality. ${ }^{1}$ Finally, TCD ultrasonography can be used only to assess velocity in the major intracranial vessels and cannot be used to address questions regarding microvascular perfusion, collateral flow, or infarcted tissue. Magnetic resonance perfusion, xenon-enhanced CT scanning, positron emission tomography, and single-photon emission computed tomography have been used to evaluate cerebral perfusion in patients with vasospasm, but they are hampered by limited availability, high costs, and the limits of patient tolerance. ${ }^{6,8}$
Computed tomography perfusion is a relatively new technique that has been studied extensively in patients with

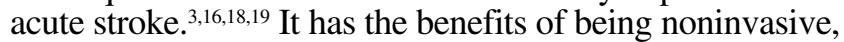
readily available in the majority of institutions, and convenient because it can be incorporated into a standard CT protocol with the addition of only a few minutes to the overall procedure time. Furthermore, unlike TCD ultrasonography, $\mathrm{CT}$ perfusion can be used to assess the hemodynamics of the cerebral microvasculature as well as the larger cerebral arteries. It also has the theoretical advantage of providing novel, sensitive information regarding regional transit times that is not available from global blood flow measurements provided by positron emission tomography and single-photon emission computed tomography.

Given the recent emergence of CT perfusion, studies on the utility and accuracy of this technology in detecting vasospasm are limited..$^{5,11,13,17}$ In the current study, we have demonstrated the usefulness of CT perfusion in the diagnosis of critically ill patients with high- and low-grade SAH. It is particularly useful in the diagnosis of unconscious patients with SAH in whom a neurological examination could not be performed. Data from CT perfusion studies can help define which of these critically ill patients should undergo DS angiography. In our study, a perfusion abnormality that suggested vasospasm was found in 15 of 23 patients. Vasospasm was confirmed in all 15 cases by using DS angiography, and the patients were treated with verapamil and/or balloon angioplasty.

Although previous studies have demonstrated the role of CT perfusion in the detection of vasospasm, almost half of them ${ }^{5,11}$ have failed to confirm the presence of vasospasm by using DS angiography, which is considered the gold standard. In our study, every CT perfusion scan was followed by cerebral angiography within 12 hours to check for the presence of vasospasm.

All CT perfusion images in our study were examined in a qualitative manner by using a combination of color maps of mean transit time, CBF, and CBV. Asymmetry or diffuse increase in mean transit time and decrease in CBF were considered to be the most important parameters regarding the diagnosis of vasospasm. In our study, CT perfusion re- 
vealed the presence of vasospasm, which was confirmed by angiography in $88 \%$ of the cases, and just as important, CT perfusion revealed the absence of vasospasm in all six cases that were confirmed as negative on DS angiography. Recently, Wintermark, et al., ${ }^{17}$ reported a similarly high detection rate for vasospasm in their series of $35 \mathrm{CT}$ perfusion scans and CT angiography studies that were followed by DS angiography studies. They concluded that a combination of a mean transit time threshold of 6.4 seconds and a qualitative assessment of CT angiograms represented the most accurate combination (93\%) for the diagnosis of vasospasm.

Our study has several limitations. First, because it was performed retrospectively, it suffers from the inherent errors of all retrospective studies. Second, the patient population and the number of CT perfusion and DS angiography studies is relatively small. Additionally, although the neuroradiologists interpreting the CT perfusion images were not aware of the results of the cerebral angiograms, they were not completely blinded to the patients' diagnoses. Finally, because DS angiography was sometimes performed based on CT perfusion findings, selection bias is likely present in our series as a confounding variable

Although CT perfusion seems to be an accurate and useful modality for detecting vasospasm, it faces some drawbacks that should be considered for every patient. The delivery of iodinated contrast agents can be problematic in patients with renal insufficiency and iodine allergy. Another disadvantage of CT perfusion is radiation exposure. Also, because perfusion parameters reported with this modality depend on the delivery of contrast material to the cerebral vessels, the results can be compromised in patients with significant cardiovascular disease. In addition, anatomical coverage is typically limited to just 4 to $8 \mathrm{~cm}$ of brain, so some lesions may be missed. Finally, interpretation of CT perfusion images can be difficult in cases in which a bone or metal artifact is encountered.

\section{Conclusions}

In our study we demonstrate that CT perfusion is a reliable modality in detecting the presence or absence of vasospasm in patients with SAH. It is noninvasive and readily available. Also, CT perfusion can aid in the decision whether a critically ill patient should undergo DS angiography for possible treatment of vasospasm. In the future, a prospective randomized study of CT perfusion is needed to assess whether this modality improves the outcome of patients with SAH.

\section{References}

1. Anonymous: Assessment: transcranial Doppler. Report of the American Academy of Neurology, Therapeutics and Technology Assessment Subcommitee. Neurology 40:680-681, 1990

2. Creissard P, Proust F: Vasospasm diagnosis: theoretical sensitivity of transcranial Doppler evaluated using 135 angiograms demonstrating vasospasm. Practical consequences. Acta Neurochir (Wien) 131:12-18, 1994

3. Eastwood JD, Lev MH, Azhari T, Lee TY, Barboriak DP, Delong DM, et al: CT perfusion scanning with deconvolution analysis: pilot study in patients with acute middle cerebral artery stroke. Radiology 222:227-236, 2002

4. Gillard JH, Antoun NM, Burnet NG, Pickard JD: Reproducibility of quantitative CT perfusion imaging. Br J Radiol 74: $552-555,2001$
5. Harrigan MR, Magnano CR, Guterman LR, Hopkins LN: Computed tomographic perfusion in the management of aneurysmal subarachnoid hemorrhage: new application of an existent technique. Neurosurgery 56:304-317, 2005

6. Hoeffner EG, Case I, Jain R, Gujar SK, Shah GV, Deveikis JP, et al: Cerebral perfusion CT: technique and clinical applications. Radiology 231:632-644, 2004.

7. Kassell NF, Sasaki T, Colohan AR, Nazar G: Cerebral vasospasm following aneurysmal subarachnoid hemorrhage. Stroke 16: 562-572, 1985

8. Koenig M, Klotz E, Luka B, Venderink DJ, Spittler JF, Heuser L: Perfusion CT of the brain: diagnostic approach for early detection of ischemic stroke. Radiology 209:85-93, 1998

9. Mayberg MR, Batjer HH, Dacey R, Diringer M, Haley EC, Heros $\mathrm{RC}$, et al: Guidelines for the management of aneurysmal subarachnoid hemorrhage. A statement for healthcare professionals from a special writing group of the Stroke Council, American Heart Association. Circulation 90:2592-2605, 1994

10. Miller J, Diringer M: Management of aneurysmal subarachnoid hemorrhage. Neurol Clin 13:451-478, 1995

11. Nabavi DG, LeBlanc LM, Baxter B, Lee DH, Fox AJ, Lownie SP, et al: Monitoring cerebral perfusion after subarachnoid hemorrhage using CT. Neuroradiology 43:7-16, 2001

12. Sloan MA, Haley EC Jr, Kassell NF, Henry ML, Stewart SR, Beskin RR, et al: Sensitivity and specificity of transcranial Doppler ultrasonography in the diagnosis of vasospasm following subarachnoid hemorrhage. Neurology 39:1514-1518, 1989

13. Sviri GE, Mesiwala AH, Lewis DH, Britz GW, Nemecek A, Newell DW, et al: Dynamic perfusion computerized tomography in cerebral vasospasm following aneurysmal subarachnoid hemorrhage: a comparison with technetium-99m-labeled ethyl cysteinate dimer-single-photon emission computerized tomography. J Neurosurg 104:404-410, 2006

14. Vora YY, Suarez-Almazor M, Steinke DE, Martin ML, Findlay JM: Role of transcranial Doppler monitoring in the diagnosis of cerebral vasospasm after subarachnoid hemorrhage. Neurosurgery 44:1237-1247, 1999

15. Weir B, Macdonald RL, Stoodley M: Etiology of cerebral vasospasm. Acta Neurochir Suppl 72:27-46, 1999

16. Wintermark M, Bogousslavsky J: Imaging of acute ischemic brain injury: the return of computed tomography. Curr Opin Neurol 16:59-63, 2003

17. Wintermark M, Ko NU, Smith WS, Liu S, Higashida RT, Dillon WP: Vasospasm after subarachnoid hemorrhage: utility of perfusion CT and CT angiography on diagnosis and management. AJNR Am J Neuroradiol 27:26-34, 2006

18. Wintermark M, Reichhart M, Cuisenaire O, Maeder P, Thiran JP, Schnyder P, et al: Comparison of admission perfusion computed tomography and qualitative diffusion- and perfusion-weighted magnetic resonance imaging in acute stroke patients. Stroke 33:2025-2031, 2002

19. Wintermark M, Reichhart M, Thiran JP, Maeder P, Chalaron M, Schnyder P, et al: Prognostic accuracy of cerebral blood flow measurement by perfusion computed tomography, at the time of emergency room admission, in acute stroke patients. Ann Neurol 51:417-432, 2002

20. Wintermark M, Thiran JP, Maeder P, Schnyder P, Meuli R: Simultaneous measurement of regional cerebral blood flow by perfusion CT and stable xenon CT: a validation study. AJNR Am J Neuroradiol 22:1018-1019, 2001

Manuscript received June 26, 2006.

Accepted in final form August 4, 2006.

Address reprint requests to: Mustafa K. Başkaya, M.D., Department of Neurological Surgery, University of WisconsinMadison, CSC K4/828, 600 Highland Avenue, Madison, Wisconsin 53792. email: m.baskaya@neurosurg.wisc.edu. 